\title{
BMJ Global Health A three-layer system to win the war against COVID-19 and invest in health systems of the future
}

\author{
Feng Zhao, Sulzhan Bali (D), Rialda Kovacevic, Jeff Weintraub
}

To cite: Zhao F, Bali S, Kovacevic R, et al. A threelayer system to win the war against COVID-19 and invest in health systems of the future. BMJ Global Health 2021;6:e007365. doi:10.1136/ bmjgh-2021-007365

Handling editor Seye Abimbola FZ and SB are joint first authors.

Received 7 September 2021 Accepted 19 November 2021

\section{Check for updates}

(C) Author(s) (or their employer(s)) 2021. Re-use permitted under CC BY-NC. No commercial re-use. See rights and permissions. Published by BMJ.

Health, Nutrition, and Population, World Bank, Washington, DC 20443, USA

Correspondence to Dr Sulzhan Bali; sbali@worldbank.org

\section{ABSTRACT}

The COVID-19 pandemic taught us many lessons, most critically that its human and economic toll would have been significantly smaller if countries had in place strong layers of defence that would have either prevented the spillover of the SARS-CoV-2 into a human population in the first place, or, failing that, contained the outbreak to avert its global spread. Further, the brunt of COVID-19 impacts on some countries considered 'most prepared' for pandemics underscored the need for an integrated approach to ensure resilience to future epidemics. Consequently, as countries plan ahead to prevent future pandemics, they should give priority to investments that transform their systems, particularly in the precrises phase, to preparedness and response through a multilayered defence. We propose a three-layered approach for post-COVID-19 investments in public health functions and service delivery, particularly at the community and precrises levels. This framework highlights the interventions that enable countries to better prevent, detect and contain epidemic threats, and that strengthen the efficient use of limited resources towards high-impact precrises systems.

\section{INTRODUCTION}

Among the many painful consequences of the COVID-19 pandemic, few will ever forget its harrowing impact on hospitals and frontline healthcare providers around the world. In the spring of 2021, for example, India's second COVID-19 wave, further complicated by the spread of the Delta variant, resulted in the pandemic's highest single-day surges of nearly 400000 infections and more than 4000 deaths. ${ }^{12}$ Across India, there were appalling scenes of hospitals spilling over with patients with COVID-19, overwhelmed and threatened the lives of medical staffs and shortages of essential materials, such as medical oxygen.

India was hardly the only country swept away by COVID-19 infections and deaths. In the first months of the outbreak, patients flooded hospitals in Wuhan, China-the epicentre of the outbreak-considerably beyond their ability to hold them. ${ }^{3}$ Not long
Summary Box

As countries draw on the lessons of COVID-19 to prevent future pandemics, they should give priority to investments that transform their systems, particularly in the pre-crises phase, to preparedness and early response through a multi-layered defense. We propose a three-layered approach for postCOVID-19 investments that integrates public health system functions and primary health care. This framework adapts the traditional, 'Prevent, Detect, and Respond' model of health security to enable prioritization, and integration of health security investments within the service delivery systems.

- The first layer is the primary foundation of pandemic defense that, among other measures, focusses on pandemic risk reduction and emphasizes primary health care systems that can monitor and detect emerging disease outbreaks at the community level.

- The second layer of defense operates primarily during the early phase of an outbreak through identification and protection of at-risk populations, scaling up of testing and contact tracing, epidemic intelligence, risk communication, and public health measures.

- Layer three includes, which would ideally be the last and rarely needed line of defense, includes surge responses,and secondary and tertiary hospital interventions that require advanced case management.

- Expenditures in first two pre-crises'layers are far smallerand are ultimately more effective in strengthening resilience to epidemics, and their secondary impacts than investments to fight a full-fledged epidemic in the final layer.

later, like a long string of burning firecrackers, those same scenes replicated themselves in big cities and small towns across literally every populated continent-even in countries such as the USA, UK and Brazil-countries previously presumed to have the strongest levels of health security. ${ }^{4-7}$ Every day, news outlets spotlighted hospitals with gurneys of patients with COVID-19 crowding hallways and emergency rooms. The demand for intensive care units (ICU) far exceeded the supply. Doctors and 
nurses worked to exhaustion and often without sufficient personal protective equipment (PPE) to fend off infection. Ventilators, essential for the most severe cases, were in short supply, and diagnostics and therapeutics were often ineffective.

At the time this article was submitted (25 October 2021), more than 243 million people had contracted COVID-19 worldwide, leading to over 4.9 million deaths. But research estimates that these official figures represent only about half of total number of deaths, leaving out a large number never reported. ${ }^{8}$ Further, the pandemic's indirect effect on nonCOVID disease prevention, detection and treatment has disrupted essential health services-leading to reductions in life expectancies, and approximately 19 million excess deaths worldwide, with regions such as Africa estimated to have excess deaths as high as $800 \% .^{910}$

Likewise, the monetary costs to reinforce hospital and advanced trauma care-expenses that no national health systems had budgeted for-have been staggering. A September 2020 Lancet Global Health article reported that those costs for low and middle-income countries were estimated at US $\$ 52$ billion (equivalent to US $\$ 8.60$ per person) in 4 weeks to provide an effective healthcare response to COVID-19. ${ }^{11}$ The International Monetary Fund found that the pandemic cost countries around the globe more than $\$ 10$ trillion in additional fiscal spending and foregone revenues, since January 2020, as well as another $\$ 6.1$ trillion in monetary support during that same time..$^{12}$ No government could morally or politically refuse to do everything possible to extinguish the pandemic's fire with whatever resources they could muster. Governments must now spend previously unimaginable sums of money to restore public health and to put their economies back on track.

Even though the crises in hospital ICUs and emergency rooms captured a large share of the media coverage around the pandemic, investing in a hospital-centric response will miss the point. Among COVID-19's many lessons is that the human and economic toll of the pandemic could have been far smaller if countries had in place strong multilayers of defence that would have either prevented the introduction of the SARS-CoV-2 into a human population in the first place, or, failing that, kept it from spreading as far and wide as it has.

The pandemic brutally illustrated how the right mixture of anthropogenic, epidemiological and demographic risk factors coupled with sociocultural factors and weak political economy can conspire to unleash the next highly contagious and deadly pandemic. Now is the time for individual governments, regions and, indeed, international entities to deploy approaches that will stop disease outbreaks long before they become epidemics or pandemics. As the Independent Panel for Pandemic Preparedness and Response concluded in its May 2021 report, the COVID-19 pandemic has been the 21st century's 'Chernobyl moment'. It argued that, unless we invest in transformed health systems that promote health security, 'we will condemn the world to successive catastrophes'. ${ }^{13}$
After years of unheeded warnings from experts that defences in most countries and regions against a disease outbreak were weak, COVID-19 should be a wake-up call to governments about the need to invest in more effective health security. This means addressing expenditures in crises, and rather upstream investments that promote and preserve human capital and avert losses during and between crises.

To this end, we propose a three-layered approach for post-COVID-19 investments that integrates public health system functions and primary healthcare (PHC). This framework adapts the traditional 'Prevent, Detect, and Respond' model of health security to enable prioritisation, and integration of health security investments within the service delivery systems. Further, this three-tier framework identifies priority investments needed at the precrises and pre-epidemic levels and at the intersection of pandemic prevention, detection and early response. This is critical, as investments in pre-epidemic interventions or preparedness have substantial returns on investments, but remain neglected. ${ }^{1415}$

\section{A PRACTICAL FRAMEWORK OF A THREE-LAYER DEFENCE SYSTEM}

This framework will fortify health systems' ability to prevent, arrest the rise of epidemics and strengthen the efficient use of limited resources towards high-impact precrises systems. The three-layered framework integrates prevention, detection and response capacities with the service delivery systems to identify three prioritised sequential layers where gaps in investment and capacity of the foundational layer will limit the progress of subsequent layers. While investment in all three layers enhances resilience, investment in foundational layers for risk reduction and community preparedness; and detection, containment and mitigation is especially critical to relieve pressure on health system as a whole.

The layers include:

- Layer 1: risk reduction - promoting prevention and community preparedness. This first layer is the primary foundation of the 'pre-crises' lines of pandemic defence. Among its core elements, this layer includes wellfunctioning, widely distributed PHC facilities, early warning systems and strong community-based surveillance systems that closely monitor and detect new disease outbreaks at the community level. It should allow direct engagement with patients to promote health behaviours and prevent and treat other conditions that make them vulnerable to emerging infectious diseases. Layer 1 will also include community and national planning (including health policies and regulations) for epidemic threats and health emergencies; strong public health institutions; well-trained health workers; a broad reach of immunisation services; strong multisectoral coordination for multisectoral interventions including strong and widely available clean water, sanitation and hygiene; health promotion and education for behaviour change; and 
One Health practices that closely examine health connections among people, animals, plants and their shared environment.

- Layer 2: detection, containment and mitigation capabilities. This layer operates primarily during the early phase of an outbreak through identification and protection of at-risk populations, scaling up of testing, isolation of suspect cases, epidemic intelligence and contact tracing, and the implementation of nonpharmaceutical interventions (NPIs) at the primary level. While detection as a function cuts across layers, timely detection is especially critical to contain an epidemic and is predicated on both stronger layer 1 as well as stronger existing laboratory capacities. It can help flatten the infection curve and reduce the number of cases that require hospitalisation. This layer counts on further investments in integrated public health functions; scaling up of mass testing; risk communications and social mobilisation; infection prevention and control (IPC) capabilities; the availability of medical professionals; and access to sufficient stocks of PPE, diagnostics, therapeutics and vaccines. It is also the layer that delivers surveillance systems that connect local, regional, national and international levels; the continuity of essential services to continue prevention and monitoring; and treatment of all other health conditions.

- Layer 3: advanced case management and surge response. The third layer of defence includes surge response interventions and secondary and tertiary hospital interventions that require advanced case management. Even though this should be the last and, ideally, rarely needed line of defence, countries must also invest in advanced case management and surge response to ensure there is a sufficient number and distribution of secondary and specialised hospitals; critical care capacity; essential equipment and supplies and stockpiles; and surge financing to quickly meet the extraordinary costs of a full-force epidemic.

The cost of strengthening preparedness and scaling testing is much lower than hospitalisation costs related to advanced treatment. ${ }^{16}$ One study estimates that the average cost of advanced case management for COVID-19 in the USA is about US $\$ 73000$ per person. ${ }^{17}$

Expenditure in precrises layers that focus on prevention, preparedness and strengthening service delivery is far smaller and ultimately more effective in preventing epidemics and their secondary impacts than investments to fight a full-fledged epidemic in the final layer (eg, building more hospital capacity, stockpiling ventilators and therapeutic drugs). ${ }^{15} 18$

The current response to COVID-19 has relied mainly on the third layer, which is both cost intensive and the least impactful of the three layers. A focus on the third layer is a reasonable response while we are in a crisis mode, but in the absence of robust precrises tiers, the long-term impact will ultimately overtax the health system. ${ }^{19}$ For an efficient post-COVID-19 response and recovery, it is crucial to focus investments on the high-impact and costefficient prehospital layers. Instead of reacting, we ought to act differently. Instead of reinventing, there is a need to modify the existing health systems' infrastructures, and create stronger linkages, and better intercomponent and intracomponent collaboration and communication.

\section{THREE LAYERS OF DEFENCE}

As figure 1 illustrates, each layer of this approach delivers a higher potential impact than the next and, therefore, warrants greater investment.

\section{Layer 1: risk reduction by promoting prevention, and community preparedness}

Layer 1 is the furthest upstream of the three layers and aims to prevent a breach of health security in the first place. If infiltration occurs, it supports the subsequent layers of defence to collectively limit the breach and mitigate the impact. This layer is therefore the foundation of an effective health security strategy, operating in relative quiet, long before a mounting crisis alerts the broader public to its importance. Expansion of this and other system layers also greatly enhances countries' ability to detect and contain epidemic threats, as well as to deliver critical services, which contribute to better individual and collective health outcomes, essential public health functions, cost savings and even broader economic and social advances. Further, investments in community engagement, PHC and community preparedness are critical to ensure community trust in the health system, to ensure adherence to public health measures and NPIs and enable access and demand for diagnostic, preventative and therapeutic interventions in an epidemic and between epidemics.

Like the hangar mechanic whose job is to ensure that planes take off and land without incident, success at this stage means non-events- the absence of disaster. Layer 1 encompasses investments in the health sector and multisectoral investments for upstream epidemic risk reduction in health and other sectors as well. This includes, for example, leveraging collaboration with a country's education sector to promote behaviour change and public health compliance; mainstreaming a One Health approach that mitigates epidemic risks at the intersection of animals, humans and the environment; and ensuring coordination across relevant sectors at local, national and regional levels.

Investments in the first layer must improve PHC systems' ability to carry out immunisation campaigns, ensure water safety and hygiene measures and provide for good nutrition and food security. These investments will drive cost efficiency because they improve preventative and promotive healthcare access.

Further, for public health to be responsive and effective, it must be anchored deeply at the local and community levels. Community preparedness involves strengthening of core capacities of disease prevention, detection and 
INVESTING TO PROMOTE HEALTH SECURITY IN THE AFTERMATH OF COVID-19

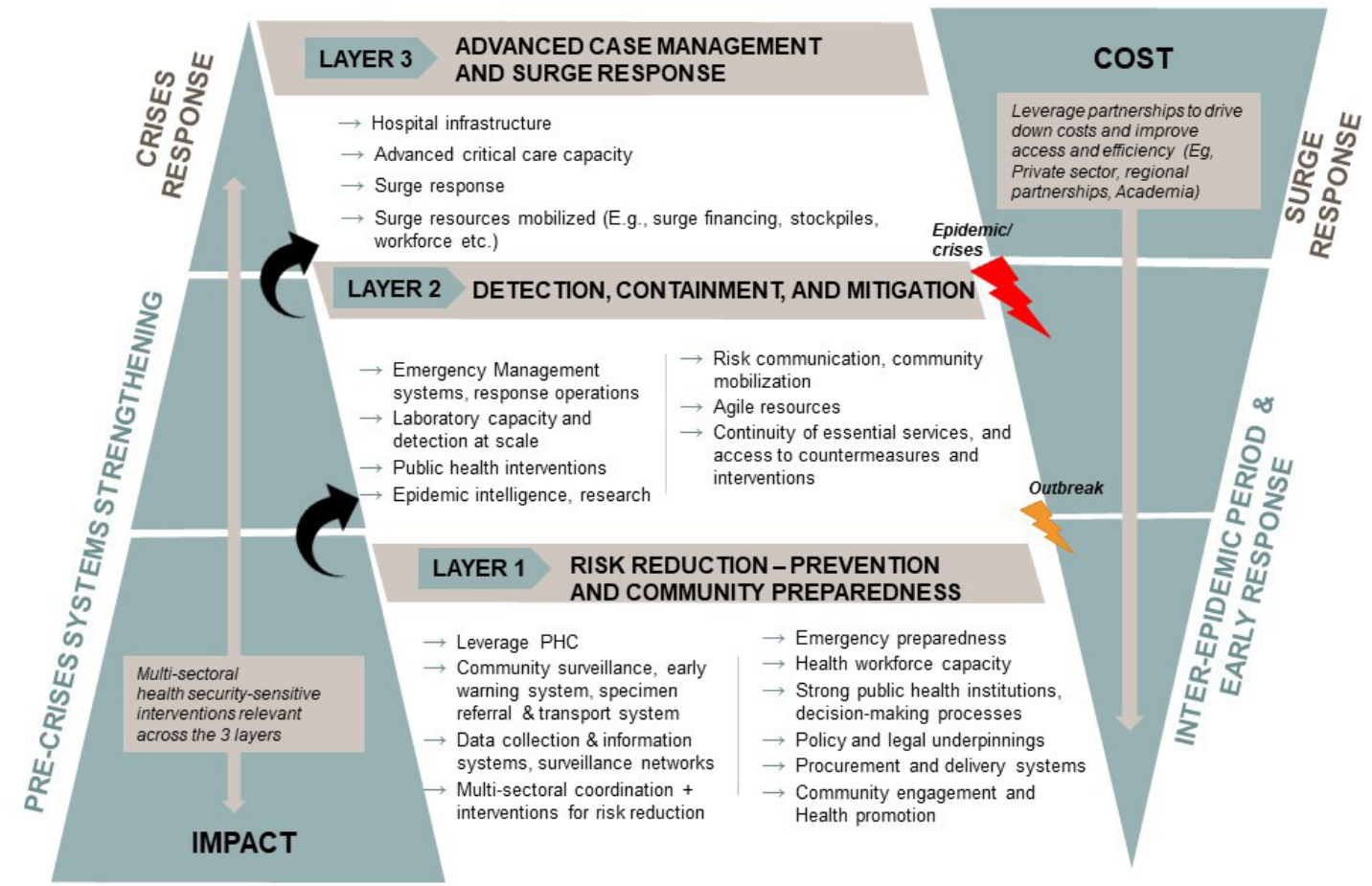

Figure 1 The three-layered framework for investing to promote health security. PHC, primary healthcare.

response readiness at the national and the most local levels. This includes firsthand patient-provider engagement, well-functioning community-based surveillance, access to preventative, diagnostic and treatment services, building the capabilities of community and primary health workers and ensuring preparedness training and access to appropriate PPE, ensuring community preparedness and contingencies.

Stronger IPC measures and access to training and PPE at the PHC level will avert infections and losses of healthcare workers. This, in turn, builds greater public trust in health systems. Without such trust, public health leaders and institutions struggle to implement their policies and interventions. As we have seen in many parts of the world, limited trust in public health has undermined countries' efforts to implement social distancing, masking and vaccination measures and to slow and control the spread of COVID-19.

Public health officials often earn and sustain trust at the local levels, even as they set national and subnational policies. Integration of more 'top-down' health policies with a 'bottoms-up' engagement from communities and providers who engage directly with patients will enhance preventative behaviour, surveillance and risk communication and inform context-specific policies through collaboration.

Layer 1 should also aim to promote multisectoral coordination, strengthen procurement and delivery systems and enact legislation and policies (such as public health acts and wildlife trade acts) that promote disease prevention, preparedness and resilience to epidemic threats.
In addition to disease prevention, this layer reflects strong community engagement and a robust communitylevel public health system, integrated within the PHC system. Experience with COVID-19 and recent epidemics suggests that a strong first layer with a community buy-in can strengthen both the 'radar screen' to detect outbreaks and the delivery of an effective response. Effective PHC is physically, financially and culturally more accessible to local communities, and as a result, able to address the disease burden that disproportionately affects poor and vulnerable populations. With a focus on health promotion and disease prevention, PHC and public health can both decrease the household expenditures for health, as well as prevent the escalation of health conditions into more complex diseases that require hospitalisation. ${ }^{13}$

Finally, stronger information systems and vital registration mechanisms improve the capture and reporting of data, which promotes evidence-based decision-making and prioritisation of resources for greater impact. Lack of early warning systems and surveillance systems can exponentially delay detection of outbreaks, ultimately increasing the cost of epidemics, and exacerbate their severity. ${ }^{20}$ A surveillance system that integrates input from public health and PHC systems at the precrises layer will be highly effective at case finding and early detection at the community level, especially in fragile and conflict areas with limited health facilities. Timely interventions can prevent small sparks from turning into megafires. ${ }^{22}$ Surveillance and detection as a function is cross-cutting; timely detection would require early warning systems, community-based surveillance systems, sample collection, 
strong sample and patient referral, and networking between national, regional and global laboratories in the first layer; and strong laboratories and laboratory capacity and epidemic intelligence in the second layer.

There are numerous examples of countries that have invested in the various elements that make up a strong first layer of disease prevention, among them:

- In 2009, an initiative to train traditional healers in northwest Uganda-an area endemic for plague and with limited access to formally trained nurses and doctors-led to development of a communitybased network of 40 traditional healers. This made it possible for 150 patient referrals to health clinics and helped a village prevent the transmission of pneumonic plague, demonstrating the impact of community preparedness in averting epidemics. Also, the formation of village health teams trained in disease surveillance and reporting has led to greater sensitisation and reporting of viral haemorrhagic diseases and served as an important link between the facility-based surveillance system and the local community. ${ }^{23}$

- Similarly, when a young herder and two students from Kenya fell ill after eating the meat of a dead cow, a Short Message Service (SMS) alert triggered by a volunteer trained in community-based surveillance under the Red Cross Society's Community Epidemic and Pandemic Preparedness Program ensured rapid notification to local health and veterinary authorities. This resulted in notification and swift action to vaccinate livestock in the vicinity and to improved community health knowledge and practices on safe disposal of animal carcasses, reporting unusual animal illness, and information on disease outbreaks. ${ }^{24}$

- In Cambodia, a scale-up of community-based surveillance, training of health workers in contact tracing and detection, and a 115 hotline sped up reporting of COVID-19 cases. It is believed to be one of the reasons for Cambodia's early success in responding to COVID-19. ${ }^{25} 26$ The country also used mathematical models to describe animal movement practices in southern Cambodia and to understand the potential spread of H5N1 revealed in a live birds market in Phnom Penh. ${ }^{27}$

\section{Layer 2: scaling detection and ensuring containment and mitigation}

With layer 1 in place, we also need a system whose primary objective is detection at scale, containment and mitigation of the disease before it spreads widely. Layer 2 essentially rests on strong first-layer components. Once an outbreak occurs, the existing structures from layer 1 can enable layer 2 to activate an early response to contain the epidemic threat. Layer 2 also seeks to leverage multisectoral partnerships and integrate inputs from public health and PHC systems to allow for timely interventions. A strong first layer can reduce frequency of outbreaks and, when an outbreak does occur, work to support layer 2 in rapid containment. Layer 1 feeds into the second layer by impacting the ability of the second layer to implement detection, containment and mitigation of the outbreak.

The COVID-19 pandemic highlighted the urgent need to invest in integrated disease surveillance and responseincluding the scale-up of contact tracing and testing, isolation of suspect cases, and ensuring implementation of public health measures and interventions to generate compliance and demand, and allowing greater access and delivery. Moreover, it has highlighted the value of technology and innovation-including telemedicine and artificial intelligence-to ensure healthcare can continue during a crisis and reduce stress on health systems when they are overly taxed. This layer helps flatten the curve of rising infection and can reduce the number of cases that require hospitalisation.

Additionally, data from COVID-19 suggest that containment strategies-such as reducing contact, shielding of elderly and social distancing measures-can drastically reduce the disease burden, avert deaths and critical care needs, thereby reducing pressure on hospitals. ${ }^{28}$

Several countries have shown how stronger containment and mitigation capabilities significantly softened the blow of COVID-19 once it arrived on their shores.

- Iceland, for instance, whose index case emerged on 28 February 2020, focused on early and extensive detection of cases by testing suspected and high-risk persons in the general population. It also relied on digital technology (Rakning C-19 app) to conduct contact tracing. In collaboration with the private sector (the biopharmaceutical company deCODE genetics), Iceland tested nearly $12 \%$ of its population, one of the highest country testing coverage rates for COVID-19 (as of 27 April 2020). Interestingly, Iceland's case fatality rate is less than $1 \%$, far lower than other high-income countries with similar age demographic distribution. ${ }^{29} \mathrm{~A}$ recent study of 37 high-income countries also revealed reduction in life expectancy in all countries-except six, which included Iceland and South Korea-underscoring the importance of containment and mitigation. ${ }^{10}$

Singapore benefited from prior experience with epidemics-namely the 2003 SARS outbreak, which spread to four healthcare facilities and led to more than 238 cases and 33 deaths, making it one of the worst affected countries. ${ }^{30}$ In 2020, aggressive contact tracing, testing, social distancing and a multipronged surveillance strategy that drew on network of primary care providers and primary health centres helped Singapore slow the spread of COVID-19. ${ }^{31}{ }^{32}$ Further, Singapore used a network of more than 800 private-public health preparedness clinics to manage the high-volume needs of testing, training of primary care physicians in IPC and response, and early case management needs. Engagement of private sector providers and the provision of free testing enabled triage and allowed the country to manage the volume of testing. However, increasing mobility, emergence of Delta strain and transmission of COVID-19 among migrant worker 
population and hidden community highlight the importance of community preparedness and rooting the early response has led to a recent surge of cases in the country, which also underscores the importance of community engagement and mobilisation..$^{33} 34$

- In Vietnam, well-developed grass-roots health systems-including primary health facilities and primary health providers-provided health education and preventative measures for COVID-19. At the same time, the country started preparing for isolation, surveillance and management for COVID-19 well in advance of the country's index case. ${ }^{35}$ The country leveraged learning from previous epidemics to scale up contact tracing and surveillance, including 12000 contact tracers and a national disease surveillance system and national public health operations centre set up in 2016. Strong contact tracing and isolation coupled with swift implementation of NPIs and use of digital media and mobile apps helped mitigate the impact of COVID-19 in Vietnam. A YouTube video created by government of Vietnam for COVID-19 prevention went viral with over 32 million views and evoked health practices through digital media. ${ }^{36}$ As of 30 January 2021, the country had fewer than 1800 COVID-19 cases and 35 deaths. However, Vietnam has experienced a rapid surge of COVID-19 cases in the summer of 2021 with the spread of Delta variant and limited vaccine coverage at the time of surge ( 6\% population had received one dose, and only $\sim 0.7 \%$ was fully vaccinated as of 2 August 2021) - underscoring the need to strengthen surveillance and ensure swift delivery of interventions such as vaccines.

- When COVID-19 arrived in Senegal among travellers returning from Europe, the country was prepared to respond. The country's health emergency operations centre, which was set up in response to the 2014-2016 West African Ebola outbreak, had been running simulations to prepare for such an outbreak for years. When COVID-19 emerged as a threat in January 2020, Senegal began assessing the country's preparedness and supporting improvements based on that assessment and relied on its strong national laboratory system. Senegal's emphasis on testing enabled it to detect early cases, and by February 2020, the Institut Pasteur de Dakar was one of two laboratories in Africa able to test for SARS-CoV-2. A series of orders to close schools and places of workshop, cancel religious festivals and institute international and regional travel restrictions was followed by a declaration of a state of emergency, which imposed a night-time curfew and a requirement to wear masks outside of the home. These efforts were supported by a comprehensive communication, and a clear communication by the government was credited with the high levels of public support for the public health measures. COVID-19 response was ranked second among 36 countries evaluated by Foreign Policy Magazine. ${ }^{37}$

\section{Layer 3: advanced case management and surge response}

The third layer of defence includes investment in surge response and secondary and tertiary hospital interventions that require advanced case management. Early detection, containment and treatment of cases through stronger precrises tiers can serve a pre-emptive and gatekeeping function and avert congestion of secondary and tertiary facilities.

As our framework explicitly points out, weak precrises systems and flimsy solutions can stress health systems during epidemics and draw outsized levels of resources in the bottomless third layer. Without the strong first and second layers, even health systems perceived to be stable will find themselves swamped with cases that outnumber their capacity.

Globally, hospitals have been facing a surge in demand for critical care and a shortage of hospital beds, tests, medical equipment like ventilators and PPE, resulting in difficult ethical choices for providers. ${ }^{38}$ Multiple analyses and real-time situations highlight that hospital systems worldwide were not prepared to cope with the astronomic surge in demand for critical care associated with COVID19. A reactive post-COVID-19 response would suggest that increasing investments in the critical care capacity of hospitals, including ICU beds, is a no-brainer. However, it may be a band-aid approach that would ignore some of the inherent systemic issues.

As the threat of COVID-19 grew in many countries and regions and overwhelmed hospitals, the tyranny of the urgent has meant that much of COVID-19 and postCOVID-19 investments would focus on secondary or tertiary-level clinical care and strengthening of critical care capacity. However, there is an urgent need to shift prioritising costly curative services at the secondary and tertiary levels over low-cost and high-impact approaches that emphasise prevention and early case detection and management, and will promote efficient utilisation of health services.

\section{CONCLUSION}

This framework has significant policy implications on future investments. While systematically investing in filling the critical gaps in all three layers is essential, policy makers must resist the tendency of investing in crises only when they occur (hence only investing in the third layer). As the COVID-19 pandemic has demonstrated, the first two layers of defence against disease outbreaks in many countries are broken. Further, the brunt of COVID-19 on some of the most 'prepared' countries highlights a need to reduce fragmentation of health security and healthcare systems, and enable prioritisation of foundational and integrated investments in risk reduction, preparedness, community resilience and service delivery systems. ${ }^{39}$ Countries that can rely on strong precrises tiers can stop outbreaks from happening, and when they occur can mitigate the overwhelming pressure on the third layer, before the crisis overwhelms the health system. While 
investment across all three layers would be critical for resilience, investments in the first two layers are foundational and will benefit public health and healthcare systems.

Subsequently, for efficient post-COVID-19 response and recovery, it is crucial to focus investments on the highimpact and cost-efficient precrises layers. ${ }^{13-16} 182140$ No country can afford sustaining the pandemic-level hospital capacity throughout normal times and in fact it is wrong to do so. The most impactful and cost-effective public investments are those that strengthen upstream interventions in a country's health system-that is, enhancements in disease prevention, preparedness (especially community preparedness) and through early detection, as well as in second-layer containment and mitigation capabilities.

These first two layers collectively can avert the congestion of secondary and tertiary facilities during epidemics. Investments in the precrises tiers will ensure efficiency and resilience of health systems and will also make it more likely that a post-COVID-19 world can address future health challenges effectively. Only doing so, there is a chance to break the 'panic and neglect' cycle.

Twitter Sulzhan Bali @sulzhan and Jeff Weintraub @JeffWeintraub

Contributors $\mathrm{FZ}$ and SB conceptualised the piece and serve as the guarantor. FZ, $\mathrm{SB}, \mathrm{RK}$ and JW provided critical inputs to the manuscript. SB and RK prepared the initial draft. SB created the figure. JW edited the piece.

Funding The authors have not declared a specific grant for this research from any funding agency in the public, commercial or not-for-profit sectors.

Disclaimer The views expressed in this article are those of the authors alone and do not represent the policies or views of the affiliated institution.

Competing interests None declared.

Patient consent for publication Not required.

Ethics approval This study does not involve human participants.

Provenance and peer review Not commissioned; externally peer reviewed.

All data relevant to the study are included in the article

Open access This is an open access article distributed in accordance with the Creative Commons Attribution Non Commercial (CC BY-NC 4.0) license, which permits others to distribute, remix, adapt, build upon this work non-commercially, and license their derivative works on different terms, provided the original work is properly cited, appropriate credit is given, any changes made indicated, and the use is non-commercial. See: http://creativecommons.org/licenses/by-nc/4.0/.

\section{ORCID iD}

Sulzhan Bali http://orcid.org/0000-0002-1146-9512

\section{REFERENCES}

1 Thiagarajan K. Why is India having a covid-19 surge? BMJ 2021;373:n1124.

2 Kapoor M, Ravi S. Making waves in India: media and the COVID-19 pandemic, 2021

3 Buckley C. 25 Days That Changed The World: How Covid-19 Slipped China's Grasp. The New York Times, 2020.

4 Abbey EJ, Khalifa BAA, Oduwole MO, et al. The global health security index is not predictive of coronavirus pandemic responses among organization for economic cooperation and development countries. PLoS One 2020;15:e0239398.

5 Haider N, Yavlinsky A, Chang Y-M, et al. The global health security index and joint external evaluation score for health preparedness are not correlated with countries' COVID-19 detection response time and mortality outcome. Epidemiol Infect 2020;148:e210.

6 Initiative NT. Johns Hopkins centre for health security and the Economist intelligence unit. Johns Hopkins Washington DC, 2019.
7 Mahajan M. Casualties of preparedness: the global health security index and COVID-19. Int $J$ Law Context 2021;17:204-14.

8 IHME. COVID-19 has caused 6.9 million deaths globally, more than double what official reports show, 2021. Available: http://www. healthdata.org/news-release/covid-19-has-caused-69-milliondeaths-globally-more-double-what-official-reports-show

9 Economist T. The pandemic's true death toll, 2021. Available: https:// www.economist.com/graphic-detail/coronavirus-excess-deathsestimates

10 Islam N, Jdanov DA, Shkolnikov VM, et al. Effects of covid-19 pandemic on life expectancy and premature mortality in 2020: time series analysis in 37 countries. BMJ 2021;375:e066768.

11 Tan-Torres Edejer T, Hanssen O, Mirelman A, et al. Projected healthcare resource needs for an effective response to COVID-19 in 73 low-income and middle-income countries: a modelling study. Lancet Glob Health 2020;8:e1372-9.

12 IMF. Fiscal Monitor Database of Country Fiscal Measures in Response to the COVID-19 Pandemic [Internet], 2021. Available: https://www.imf.org/en/Topics/imf-and-covid19/Fiscal-PoliciesDatabase-in-Response-to-COVID-19

13 Sirleaf EJ, Clark H. Report of the independent panel for pandemic preparedness and response: making COVID-19 the last pandemic. Lancet 2021;398:101-3.

14 Burwell SM. Improving pandemic preparedness: lessons from covid-19. Council on Foreign Relations, 2020.

15 World Bank. From panic and neglect to investing in health security: financing pandemic preparedness at a national level, 2017.

16 Sousa-Pinto B, Fonseca JA, Costa-Pereira A. Is scaling-up COVID-19 testing cost-saving? medRxiv 2020.

17 Hoffower $\mathrm{H}$. If you're hospitalized with COVID-19 and don't have insurance, you'll likely owe $\$ 73,000$. One chart shows how broken the system is for the most vulnerable. Business Insider, 2020.

18 GPMB. From worlds apart to a world prepared: global preparedness monitoring board report, 2021.

19 Waldman A. Are hospitals near me ready for coronavirus? here are nine different scenarios, 2020. Available: https://projects.propublica. org/graphics/covid-hospitals

20 Kieny M-P, Evans DB, Schmets G, et al. Health-system resilience: reflections on the Ebola crisis in Western Africa. Bull World Health Organ 2014;92:850.

21 World Bank. Lessons learned in financing rapid response to recent epidemics in West and central Africa: a qualitative study. World Bank, 2019.

22 Ratnayake R, Tammaro M, Tiffany A, et al. People-centred surveillance: a narrative review of community-based surveillance among crisis-affected populations. Lancet Planet Health 2020;4:e483-95.

23 National Academies of Sciences, E. and Medicine. Global health risk framework: resilient and sustainable health systems to respond to global infectious disease outbreaks: workshop summary. National Academies Press, 2016.

24 Epidemics that didn't Happen | Case Study: ANTHRAX in Kenya 2020, 2021. Available: https://preventepidemics.org/epidemics-thatdidnt-happen/anthrax/

25 Crawley A. Surveillance technology in Thailand, Cambodia, and Tanzania: case study from ending pandemics, 2021. Available: https://www.exemplars.health/emerging-topics/epidemicpreparedness-and-response/surveillance-technology-endingpandemics-case-study

26 Nit B, Samy AL, Tan SL, et al. Understanding the slow COVID-19 trajectory of Cambodia. . Public Health in Practice, 2021: 2. 100073 .

27 UNISDR. Using science for disaster risk reduction, 2013.

28 Branas CCet al. Flattening the curve before it flattens us: Hospital critical care capacity limits and mortality from novel coronavirus (SARS-CoV2) cases in US counties. medRxiv 2020.

29 Coronavirus pandemic (COVID-19), 2021. Available: https:// ourworldindata.org/coronavirus/country/iceland

30 Goh K-T, Cutter J, Heng B-H, et al. Epidemiology and control of SARS in Singapore. Ann Acad Med Singap 2006;35:301-16.

31 Lee VJ, Chiew CJ, Khong WX. Interrupting transmission of COVID-19: lessons from containment efforts in Singapore. J Travel Med 2020;27.

32 Lim WH, Wong WM. COVID-19: notes from the front line, Singapore's primary health care perspective. Ann Fam Med 2020;18:259-61.

33 Yip W, Ge L, Ho AHY, et al. Building community resilience beyond COVID-19: the Singapore way. Lancet Reg Health West Pac 2021;7:100091

34 Tan EY, Albarazi D, Saw YE, et al. Confidence in government and rumors amongst migrant worker men involved in dormitory 
outbreaks of COVID-19: a cross-sectional survey. J Migr Health 2021;4:100069.

35 Epidemics that didn't Happen| Case Study: COVID-19 In Vietnam, 2021. Available: https://preventepidemics.org/epidemics-that-didnthappen/covid-19-vietnam/

36 Ha BTT, Ngoc Quang L, Mirzoev T, et al. Combating the COVID-19 epidemic: experiences from Vietnam. Int $J$ Environ Res Public Health 2020;17:3125.

37 Epidemics that didn't Happen| Case Study: COVID-19 In Senegal, 2021. Available: https://preventepidemics.org/epidemics-that-didnthappen/covid-19-senegal/
38 Shortland N. Doctors are making life-and-death choices over coronavirus patients - it could have long-term consequences for them, 2020. Available: http://theconversation.com/doctors-aremaking-life-and-death-choices-over-coronavirus-patients-it-couldhave-long-term-consequences-for-them-134728

39 Lal A, Erondu NA, Heymann DL, et al. Fragmented health systems in COVID-19: rectifying the misalignment between global health security and universal health coverage. Lancet 2021;397:61-67.

$40 \mathrm{WHO}$. Building the economic case for primary health care: a scoping review, 2018. 